\section{Haplotypes, sub-haplotypes and geographical distribution in Omani patients with sickle cell disease}

\author{
Suha Mustafa Hassan, ${ }^{1,2}$ \\ Muhanna Al Muslahi, ${ }^{2}$ Muna Al Riyami, ${ }^{2}$ \\ Abeer Al Balushi, ${ }^{2}$ Egbert Bakker, ${ }^{3}$ \\ Cornelis L. Harteveld, ${ }^{3}$ Piero C. Giordano ${ }^{3}$ \\ 1 Molecular Genetic Laboratory, Genetics \\ Centre, Muscat, Sultanate of Oman; \\ ${ }^{2}$ Royal Hospital, Muscat, Sultanate \\ of Oman; ${ }^{3}$ The Reference \\ Hemoglobinopathies Laboratory, \\ Department of Human and Clinical \\ Genetics, Leiden University Medical \\ Centre, Leiden, The Netherlands
}

\section{Abstract}

Despite the fact that patients homozygous for the sickle cell disease (SCD) mutation have an identical genotype, the severity of the disease can be extremely variable. The hemoglobin $(\mathrm{Hb}) \mathrm{S}$ mutation has been described on five different haplotypes with different clinical expression. Identifying the genotypes, haplotypes and sub-haplotypes of the $\beta$ gene cluster in Oman needs to be studied in more details to establish a correlation between the genotype/ haplotype and phenotype diversity observed in SCD patients for prognostic purposes, accurate diagnosis and thus planning for the best tailored treatment. We have investigated $125 \mathrm{HbS}$ homozygotes from different parts of Oman and determined their haplotypes and sub-haplotypes and correlated this to the hematological and clinical expression. We have found 11 haplotype combinations differently distributed in the country, with the Asian/Asian HbS haplotype being the most predominant. Sub-haplotypes was only found among patients with CAR/OmanI haplotype. As expected, the correlation between haplotypes, sub-haplotypes and disease severity was mainly associated with $\mathrm{HbF}$ expression. Our study on haplotype/phenotype correlation has shown which major haplotypes occur in the different regions of Oman. Furthermore, neither the haplotype or sub-haplotype nor the $\mathrm{HbF}$ alone appeared to be fully associable with the variable clinical phenotypes. External factors do occur and are associated with the expression of the disease.

\section{Introduction}

Sickle cell disease (SCD) is one of the most common autosomal recessive disorders in human and was first described by Herrick in $1910 .{ }^{1}$ The disease is caused by a single nucleotide transversion at codon $6 \mathrm{GAG}>\mathrm{GTG}$ (HBB:c.20A>T) (NM_000518.4) of the $\beta$ globin gene resulting into the commonest hemoglobin variant worldwide $(\mathrm{HbS})$ characterized by the single Glu®Val amino acid substitution at position $\beta 6 .^{2}$ Despite the fact that all patients homozygous for the $\mathrm{HbS}$ allele have an identical genotype, the severity of the disease can be extremely variable among affected subjects. ${ }^{3}$ The disease may manifest with full blow severity, with chronic and acute infarctions in organs and tissues causing excruciating pain episodes (crisis), brain infarctions, splenic infarction, massive hemolytic events and acute chest syndrome with risk of premature death. Other cases however, may present with milder symptoms and the variability is mainly associated with the haplotype, sub-haplotype, $\alpha$ thalassemia ${ }^{4}$ and the presence of fetal hemoglobin ( $\mathrm{HbF})(\alpha 2 \gamma 2),{ }^{5}$ which may be attributed to the coinheritance of Xmn-I polymorphism. This marker is important for early prevention or reduction of morbidity in SCD patients treated with hydroxyurea therapy ${ }^{6}$ or to decide whether or not bone marrow transplantation should be considered. ${ }^{7}$ The HbS mutation has been described on five distinct haplotypes based on the presence or absence of the 5 different restriction enzyme sites in the $\beta$-globin gene cluster located on the 5 ' and two restriction enzyme sites on the 3 ' sides of the $\beta$ gene (Table 1). ${ }^{8-11}$ These haplotypes are known as Benin, Bantu or Central African Republic (CAR), Senegal, Cameroon and Asian. ${ }^{8}$ The first four are African haplotypes, named after their origin and ethnic group ${ }^{9}$ while the last was described in Central India and Saudi Arabia. ${ }^{10}$ It has been previously reported that the CAR haplotype is usually associated with a more severe disease when compared with the intermediate phenotype of the Benin haplotype and to the milder conditions associated with the Senegal and Asian haplotypes. ${ }^{11}$

Analysis of the polymorphic sites of the $\beta$ genes cluster is of genetic, anthropologic and clinical interest, and it can also be used to predict the prognosis of the disease and to plan a tailored treatment.

The work reported here involves the investigation of a serial of polymorphic sites (SNP's) within the $\beta$ globin gene cluster to identify the $\mathrm{HbS}$ haplotype of Omani patients. For this, we have selected 125 Omani SCD patients with homozygous $\mathrm{HbS}$ conditions and compared their haplotype with hematological and clinical data. Moreover, to look if there are any subhaplotypes within each known haplotype that might be associated with the clinical differences seen in patients with the same HbS haplotype, extra SNP's, in addition to the common ones have been studied as suggested by
Correspondence: Suha Mustafa Hassan, Hemoglobinopathies Laboratory, Molecular Genetics, Genetics Center, Ministry of Health, Muscat, Oman.

E-mail: suha_85@hotmail.com

Key words: Sickle cell disease; genotype; haplotype; sub-haplotype; Oman.

Conflict of interest: the authors declare no conflict of interest.

Received for publication: 2 October 2014. Revision received: 12 February 2015.

Accepted for publication: 6 March 2015.

This work is licensed under a Creative Commons Attribution 3.0 License (by-nc 3.0).

(C) Copyright S.M. Hassan et al., 2015

Licensee PAGEPress, Italy

Thalassemia Reports 2015; 5:4739

doi:10.4081/thal.2015.4739

Marion et al. ${ }^{12}$ Looking for correlations with the clinical phenotypes, we have also charted the distribution of the $\beta$ gene haplotypes in different regions of the country and characterized the different haplotypes and sub-haplotypes using advanced molecular technologies.

\section{Materials and Methods}

\section{Subjects}

We have collected, with signed consent of patients and families, blood samples from a cohort of $125 \mathrm{SCD}$ patients, whether admitted or following up in one of the Ministry of Health Hospitals in Oman. Gender distribution was; 84 males (45\%) and 103 females (55\%). The ages of the subjects were on average $36 \pm 5$ years.

\section{Strategy}

Cation-exchange high performance liquid chromatography was performed on all samples on either D-10 (short and extended programs) device and/or Variant II (Bio-Rad Laboratories, Hercules, CA, USA) to measure the rate of $\mathrm{HbF} / \mathrm{HbS}^{13}$ in absence or before blood transfusion. DNA was extracted using the Qiagen kit according to the manufacturer instruction as previously described. ${ }^{14} 47$ SNP's, covering 13 sites in the $\beta$-globin gene cluster that are known to be variable and informative were analysed (Figure 1). This was carried out by an asymmetric polymerase chain reaction $(\mathrm{PCR})^{12}$ and analysed by melting curve analysis (MCA). ${ }^{15}$ The common haplotyping of the $\beta$ globin gene cluster was determined according to the presence (+) or absence (-) of a composition of SNP's corresponding to the traditional 
five 5' known polymorphic restriction endonulease sites (F1, F2 (SNP 2), F3 (SNP 2), F4 and F5). ${ }^{12}$ The remaining 42 SNP's were used to look for sub-haplotypes to find out if there are nucleotide variations within similar haplotypes that might be associated with the phenotypical differences observed.

\section{Genotyping procedures}

Genotypes of PCR products were determined using the Light Scanner (Idaho Technologies Inc., Salt Lake City, UT, USA) after performing asymmetric PCR with LCGreen fluorescent DNA dye and unlabeled oligonucleotide probes. ${ }^{12}$ The PCR was performed asymmetrically so that the strand complementary to the probe is produced in excess, allowing probe annealing at the SNP site. The primers were designed to yield a product not larger than 200 bp as it is the optimal length for accurate scanning and genotyping with MCA. A fluorescent dye (LC Green plus) that emits light in the presence of double stranded DNA was added to the reaction and as the temperature increases, the fluorescence decreases as the dsDNA melts out; this produces a characteristic melting curve. ${ }^{15}$ Unlabeled oligonucleotide probes were designed and used to genotype targeted sequence variations. These probes increase specificity of the melting reaction as it decreases the size of the product that is melted. Therefore, the probe is designed to anneal to either the wild type or the mutant allele; the characteristic melting curves identify the genotype of each sample. ${ }^{16}$ The shape of the PCR amplicon melting curve reveals the presence or absence of the SNP in comparison to the wild type sequence, allowing clear recognition and genotyping. ${ }^{15}$ The haplotype/sub-haplotype is then drawn from the obtained genotypes. In case of homozygosity for all markers, the haplotype can be defined. In the case of a single SNP difference, two distinct haplotypes can be defined. In case of two or more SNP differences, the most likely/frequent haplotype in the population is defined. SNP's that were doubtful were sequenced by Sanger sequencing for confirmation.

\section{Phenotype classification}

In order to define disease severity, a number of well-defined clinical parameters were analysed: hemoglobin level, frequency of transfusion which is based on episodes of acute hemolysis (e.g. pulmonary hypertension, jaundice, gallstones, splenic crisis), number of annual hospitalization, frequency of vaso-occlusive crisis, splenectomy (indicated by acute splenic sequestration and chronic hypersplenism), acute chest syndrome (ACS), body pain (e.g. abdomen, chest, bones, joints, episode of dactylitis) and records of any major organ damage such as heart and liver (Table 2). The severity of disease expression was then correlated with the haplotype and hematological parameter readings of $\mathrm{HbF}$ and $\mathrm{HbS}$.

Table 1. The five most common haplotypes named after their origin and ethnic group are summarised and compared phenotypically.

\begin{tabular}{lcc} 
Haplotype & Origin & Phenotype \\
Benin & African & Intermediate \\
Bantu or Central African Republic & African & Severe \\
\hline Senegal & African & Mild \\
Cameroon & African & Intermediate \\
\hline Asian or Arab/Indian & Central India and Saudi Arabia & Mild \\
\hline
\end{tabular}

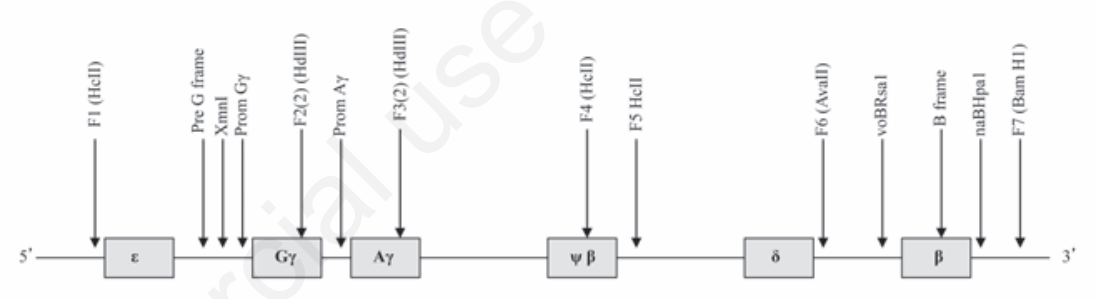

Figure 1. Schematic representation of the $\beta$-globin gene cluster. The arrows indicate the locations of the 13 different regions that contain the 47 serial of polymorphic sites (SNPs), including the 5 sites screened for the traditional haplotypes. The extra 42 SNPs are explained as follow: F1 contains 1 SNP in the 5' region of the $\varepsilon$-gene. The preGframe in the 5' region of the G $\gamma$-gene contains 5 SNPs that are linked and only occur in a limited number of combinations. The promoter regions of the $\gamma$-genes; promG $\gamma$ and promA $\gamma$ contain 10 SNPs each, among which are the non-deletional HPFH point mutations. Also among the $\mathrm{G} \gamma$ promoter is the Xmn-I site, which is known to cause continued expression of $\mathrm{HbF}$ during adult life in case of erythropoietic stress. F2, in intron 2 of the $\mathrm{G} \gamma$-gene, and F3, in intron 2 of the A $\gamma$-gene both contain 4 SNPs. F4 contains 1 SNP in the pseudo $\beta$-gene. F5 contains 1 SNP in the 3' region of the pseudo $\beta$-gene. F6 contains 1 SNP in the 3' region of the $\delta$-gene. The voBRsal fragment in the 5' region of the $\beta$-gene contains 5 SNPs. This fragment also contains a very polymorphic (AT)xTy repeat located after SNP 1 that includes SNP 2 . The $\beta$-frame in the $\beta$-gene contains 3 linked SNPs that only occur in a limited number of combinations. The naBHpal fragment in the 3' region of the $\beta$ gene contains 1 SNP. F7 in the 3' region of the $\beta$-gene contains 1 SNP. In addition, the $\mathrm{XmnI}$ site has also been indicated in the figure.

Table 2. Classification criteria for the 125 homozygous $\mathrm{HbS} / \mathrm{S}$ patients to assess sickle cell disease severity into mild, intermediate and severe.

\begin{tabular}{|c|c|c|c|}
\hline Clinical classification of SCD patients $(n=125)$ & Mild $(n=46)$ & Intermediate $(\mathrm{n}=\mathbf{3 1})$ & Severe $(n=48)$ \\
\hline Hemoglobin level & $\uparrow 9.1$ & $\downarrow 8.5$ & $\downarrow 8$ \\
\hline Transfusions/hemapheresis & Not required & Occasional & Frequent \\
\hline Hospitalizations per year & $1-2$ & $2-4$ & $>5$ \\
\hline Crisis frequency & $\downarrow 3$ per year & $\uparrow 3$ per year & $\uparrow 6$ per year \\
\hline Splenectomy & Yes & Yes & Yes \\
\hline Acute chest syndrome & No & No & Yes \\
\hline Body pain & Mild & Moderate & Intense \\
\hline Severe organ damage & No & No & Yes \\
\hline
\end{tabular}


Asian/Asian haplotype, mostly were presented with a mild disease and none with a severe form. Conversely, Benin/Benin ranged from severe to intermediate while none presented with the mild form. Among the Asian/0manI haplotype, the percentage was equal between mild and intermediate. The majority of homozygous CAR and compound heterozygous CAR/OmanI $\beta$ cluster had a severe clinical profile. Finally, the phenotype of Asian/CAR haplotype ranged from mild to intermediate and even severe cases. Data are summarized in Table 3.

melting curve analysis. The homozyous Asian haplotype was the most predominant (37.6\%). The second most prevalent was the homozygous Benin haplotype (20.0\%). The remaining haplotypes were distributed as follow: compound heterozygous Asian/0manI (11.2\%), homozygous CAR (10.4\%), compound heterozygous CAR/0manI (10.4\%), CAR/Asian (6.4\%), homozygous Omani (0.8\%), compound heterozygous of Senega//OmanI (0.8\%), Benin/OmanII (0.8\%), Benin/0manIII (0.8\%) and finally Asian/0manIV $(0.8 \%)$. Data are summarized in Table 3.

\section{Clinical severity}

The phenotypes of the patients were classified into mild, intermediate and severe based on the described criteria (Table 2). Data were obtained from patient's medical records anonymously provided by the doctor. Among the

\section{Hematological data}

The $\mathrm{Hb} F$ value correlates with the different haplotypes, giving a direct indication of the disease severity. The average hemoglobin values, $\mathrm{HbF}$ and $\mathrm{HbS}$ percentage of each haplotype are summarized in Table 4. The Asian haplotype, being associated with a mild phenotype, presented with the highest expression of $\mathrm{HbF} \%$ and the lowest $\mathrm{HbS} \%$ when compared to the other haplotypes. Patients with Asian/OmanI haplotype had a mild to intermediate phenotype. This can be attributed to the elevated expression of $\mathrm{HbF} \%$ (Table 4). Conversely, the homozygous CAR and compound heterozygous CAR/OmanI haplotypes had the most severe clinical picture with the lowest $\mathrm{Hb} \mathrm{F}$ values (Table 4).

\section{Geographical distribution}

The overall distribution of each haplotype in our SCD patients in the different regions of Oman is presented in percentages in Table 5 . The occurrence frequency of each haplotype in a particular region is depicted in Figure 2. In Musandam, CAR/Asian is the only haplotype found. In Batinah, Asian/0manI is the most abundant. In Muscat, CAR/OmanI is found at the highest frequency and in Sharqiya, the Asian haplotype was the most prevalent. In Dhakhiliya, Benin was the major haplotype found and finally in Dhahira, the CAR haplotype was the most prominent. Data are summarized in Figure 2.

\section{Sub-haplotypes}

Based on additional SNP's, a subdivision could be made from the original five $\mathrm{HbS}$ haplotypes. Trying to find a molecular explanation for the different phenotypes seen within similar basic haplotypes, sub-haplotypes were determined by looking at a total of 42 SNP's in all the 125 homozygous $\mathrm{HbS}$ patients. Out of the 42 SNP's, only 15 SNP's were found modifying the 11 identified haplotypes. However, no sub-haplotypes were found to be associated with a specific haplotype except for the CAR/OmanI that showed nucleotide variations at the G- $\gamma$ (SNP1) (SNP position: 52329795232984) located in the $\mathrm{G}-\gamma$ promoter region

Table 3. Summary of the genotypes from which the 11 haplotypes were determined in the Omani HbS/S homozygous patients using the five 5' traditional sites. The Xmn-I genotype and percentages of mild, intermediate and severe cases within each haplotype is also indicated.

\begin{tabular}{|c|c|c|c|c|c|c|c|c|c|c|c|c|}
\hline Haplotype & $\begin{array}{c}\text { HcII }\left(5^{\prime} \varepsilon\right) \\
\text { F1 } \\
\left.\left(\mathrm{A}:+/ \mathrm{C}^{\prime}-\right)^{-}\right)\end{array}$ & $\begin{array}{c}\text { Xmn-I } \\
(C:-/ \mathbb{I}+)\end{array}$ & $\begin{array}{c}\text { Hd III }(\mathrm{G} \gamma) \\
\text { F2(2) } \\
\mathrm{G}:-/ \mathrm{I}:+)\end{array}$ & $\begin{array}{l}\text { Hd III }(A \gamma) \\
\text { F3(2) } \\
(\mathrm{G}:-/ \mathrm{T}:+)\end{array}$ & $\begin{array}{c}\text { Hc II }(\Psi \beta) \\
\text { F4 } \\
(\mathrm{G}:-/ \mathrm{A}:+)\end{array}$ & $\begin{array}{c}\text { Hc II }(3 \Psi \beta) \\
\text { F5 } \\
(\mathrm{G}:-/ \mathrm{A}:+)\end{array}$ & $\begin{array}{c}\text { Ava II }(\beta) \\
\text { F6 } \\
\left(G:-/ C_{s+}\right)\end{array}$ & $\begin{array}{c}\text { Bam HI (3’ } \beta) \\
\text { F7 } \\
(\mathrm{C}, \mathrm{T}:-/ \mathrm{A}:+)\end{array}$ & Total & $\begin{array}{l}\text { Mild } \\
\%\end{array}$ & $\begin{array}{c}\text { Intermediate } \\
\%\end{array}$ & $\begin{array}{c}\text { Severe } \\
\%\end{array}$ \\
\hline Asian/Asian & $\mathrm{AA}$ & TT & TT & $\mathrm{GG}$ & $\mathrm{AA}$ & $\mathrm{AA}$ & $\mathrm{GG}$ & $\mathrm{C}, \mathrm{T} / \mathrm{C}, \mathrm{T}$ & 47 & 78.7 & 21.3 & 0 \\
\hline Benin/Benin & $\mathrm{CC}$ & $\mathrm{CC}$ & GG & GG & GG & $\mathrm{AA}$ & GG & $\mathrm{AA}$ & 25 & 0 & 36 & 64 \\
\hline Asian/Oman I & $\mathrm{AC}$ & $\mathrm{TC}$ & TT & GG & $\mathrm{GA}$ & $\mathrm{AA}$ & GG & $\mathrm{A} / \mathrm{C}, \mathrm{T}$ & 14 & 50 & 50 & 0 \\
\hline CAR/CAR & $\mathrm{CC}$ & $\mathrm{CC}$ & TT & GG & GG & GG & $\mathrm{GG}$ & $\mathrm{AA}$ & 13 & 0 & 7.7 & 92.3 \\
\hline CAR/Oman I & $\mathrm{CC}$ & $\mathrm{CC}$ & TT & GG & GG & GA & GG & $\mathrm{AA}$ & 13 & 0 & 25 & 75 \\
\hline CAR/Asian & $\mathrm{AC}$ & $\mathrm{TC}$ & TT & GG & $\mathrm{GA}$ & GA & GG & $\mathrm{A} / \mathrm{C}, \mathrm{T}$ & 8 & 12.5 & 37.5 & 50 \\
\hline Oman I/Oman I & $\mathrm{CC}$ & $\mathrm{CC}$ & TT & GG & GG & $\mathrm{AA}$ & GG & $\mathrm{AA}$ & 1 & 0 & 0 & 100 \\
\hline Senegal/OmanI & $\mathrm{CC}$ & $\mathrm{TC}$ & TT & GG & $\mathrm{GA}$ & $\mathrm{AA}$ & GG & $\mathrm{AA}$ & 1 & 0 & 0 & 100 \\
\hline Asian/Oman II & $\mathrm{AA}$ & $\mathrm{TC}$ & TG & $\mathrm{GG}$ & GA & GA & CG & $\mathrm{C}, \mathrm{T} / \mathrm{C}, \mathrm{T}$ & 1 & 0 & 0 & 100 \\
\hline Benin/Oman III & $\mathrm{AC}$ & $\mathrm{CC}$ & TG & GG & GG & GA & CG & $\mathrm{AA}$ & 1 & 0 & 0 & 100 \\
\hline Benin/Oman IV & $\mathrm{CC}$ & $\mathrm{CC}$ & GG & GG & $\mathrm{GG}$ & GA & $\mathrm{GG}$ & $\mathrm{AA}$ & 1 & 100 & 0 & 0 \\
\hline
\end{tabular}

Table 4. Summary of hematological data (average) for each haplotype.

\begin{tabular}{lcccccc} 
& Asian/Asian & Benin/Benin & Asian/OmanI & CAR/CAR & CAR/OmanI & CAR/Asian \\
$\mathrm{Hb}(\mathrm{g} / \mathrm{dL})$ & $9.5 \pm 1.3$ & $9.3 \pm 1.7$ & $10.3 \pm 1.9$ & $7.5 \pm 1.1$ & $8.7 \pm 1.2$ & $9.1 \pm 1.4$ \\
$\mathrm{HbF}(\%)$ & $18.2 \% \pm 5.9$ & $6 \% \pm 3.1$ & $14.2 \% \pm 5.5$ & $5.9 \% \pm 4.9$ & $5 \% \pm 3.5$ & $12.6 \% \pm 5.4$ \\
\hline $\mathrm{HbS}(\%)$ & $72.3 \% \pm 6.9$ & $80.3 \% \pm 5.7$ & $75.2 \% \pm 7.6$ & $80.7 \% \pm 7.7$ & $78.5 \% \pm 9.1$ & $78.3 \% \pm 7.9$ \\
\hline
\end{tabular}


from which three different sub-haplotypes were defined ( $a, b$ and $c$ ) (Table 6 ). This site might be associated with the different clinical expression observed in CAR/OmanI patients. The patient with sub haplotype (CAR/0manI c) presented with a very severe clinical manifestations and no improvement was seen even after doubling the dosage of hydroxyurea. This patient appears to be homozygous for the wild type 6 nucleotides (CTTTAA) at the G- $\gamma$ promoter. On the other hand, another patient with sub haplotype (CAR/OmanI - b) was homozygous for the deletional mutation of the 6 nucleotides at the same position and had a milder clinical presentation and was not taking hydroxyurea consistently as the patient was feeling better after a short period of being on the drug. The remaining patients with subhaplotypes (CAR/OmanI - a) had the compound heterozygous composition of CTTAA/ 6 nt del at the $\mathrm{G}-\gamma$ promoter region, needed higher than the average dose of hydroxyurea in order to observe a reduction of the severe phenotype. Although more case studies are needed to confirm our hypothesis, our data allow us to assume that carrying the 6 -nucleotide deletion might be beneficial to CAR/OmanI patients for better response to hydroxyurea.

\section{Discussion}

\section{Haplotypes distribution and dis- ease severity}

The Omani populations are known for their high incidence of hemoglobinopathies, including $\alpha$ and $\beta$-thalassemia as well as sickle cell disease. ${ }^{14}$ As mentioned above, patients with SCD present with a variable clinical picture ranging from severe to very mild forms, where haplotypes have been found to be associated with the severity of the disease. ${ }^{17,18}$ In Daar et $a l$, a study conducted in Oman in 2000 on 52 $\mathrm{HbS} / \mathrm{S}$ individuals, it was found that the Benin/Benin haplotype was the most prevalent and twice more frequent than the Asian/Asian. ${ }^{19}$ In the present study however, the Asian/Asian haplotype was the most prominent while Benin/Benin was the second in rank (Table 3). The reason for the higher per- centages of Benin haplotype in Daar et al., might be due to a selection among patients attending the Sultan Qaboos University Hospital which are mainly from the Dhakhiliya region and based on our findings, the Benin haplotype has been observed to be present at a high rate in this region (Figure 2). Although the effect of these haplotypes on the phenotypes is clearly correlating with the $\mathrm{HbF}$

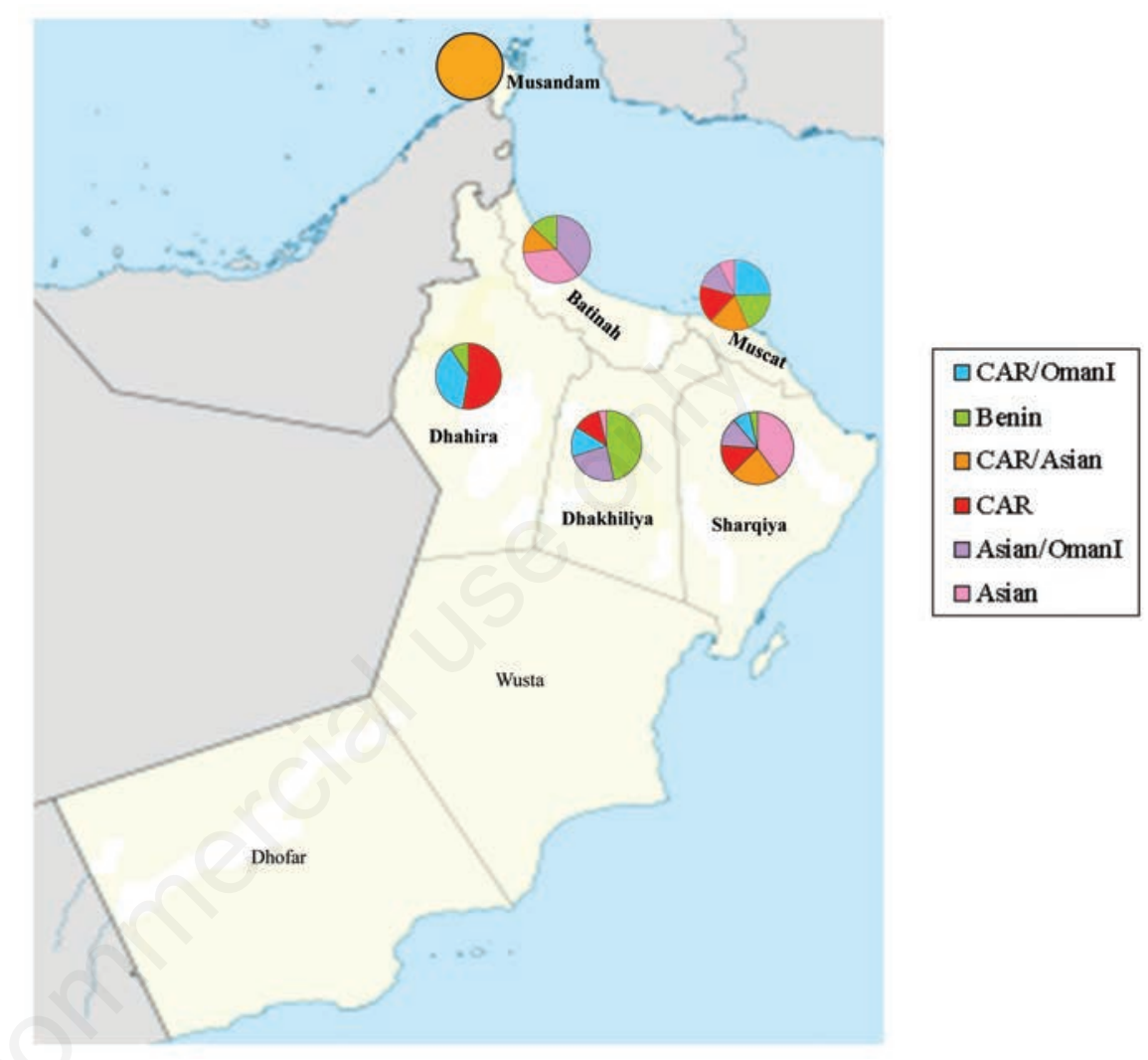

Figure 2. The geographical distribution of the $\beta$ globin gene cluster haplotypes within Oman.

Table 5. The frequency distribution of the 6 most common haplotypes in 6 different regions in Oman.

\begin{tabular}{|c|c|c|c|c|c|c|}
\hline RegionVaplotype & Asian/Asian & Benin/Benin & Asian/0manI & CAR/CAR & CAR/OmanI & CAR/Asian \\
\hline Musandam & - & - & - & - & - & $12.5 \%$ \\
\hline Batina & $31.9 \%$ & $12 \%$ & $35.7 \%$ & - & - & $12.5 \%$ \\
\hline Muscat & $21.3 \%$ & $52 \%$ & $35.7 \%$ & $46.2 \%$ & $66.7 \%$ & $50 \%$ \\
\hline Sharqiya & $44.7 \%$ & $4 \%$ & $14.3 \%$ & $15.3 \%$ & $8.3 \%$ & $25 \%$ \\
\hline Dhakhiliya & $2.1 \%$ & $28 \%$ & $14.3 \%$ & $7.7 \%$ & $8.3 \%$ & - \\
\hline Dhahira & - & $4 \%$ & - & $23.1 \%$ & $16.7 \%$ & - \\
\hline Total \% & 100 & 100 & 100 & 100 & 100 & 100 \\
\hline
\end{tabular}

Table 6. The different genotypes observed at the G- $\gamma$ promoter (SNP1) among CAR/OmanI patients, suggesting the existence of three different sub-haplotypes $(a, b$ and $c)$.

\begin{tabular}{lccc} 
CAR/Omanl sub-haplotype & G $\gamma(1)$ & No. of patients & Response to HU \\
a & CTTTAA/6nt del & 11 & Mild \\
b & 6nt del/6nt del & 1 & Positive \\
\hline $\mathrm{c}$ & CTTTAA/CTTTAA & 1 & None \\
\hline
\end{tabular}


expression, disease severity remains variable within the same haplotypes and more molecular and external factors need to be taken into consideration. The high frequency of $\alpha$-thalassemia reported in the Omani population ${ }^{14}$ is another modulating factor influencing the clinical outcome of the disease. The effect of $\alpha$ thalassemia on the clinical expression of SCD is under evaluation in our cohort and will be presented in a following paper.

\section{Gene flow}

The distribution of the $\mathrm{HbS}$ haplotypes in Oman is explained by the historical migrations from Zanzibar and India. The presence of the Asian haplotype can be attributed to ancient migrations and to centuries of trade with India and Pakistan. Contacts with East Africa, Zanzibar and Mombasa, explain the presence of the Benin and CAR haplotypes. ${ }^{19}$ Muscat, being the capital, had the widest diversity of different haplotypes. This reflects more recent migrations of native people from the interior to the capital seeking for jobs and better lives. The Asian haplotype was highest in Sharqiya and Batinah and these regions are known to have SCD patients with a mild clinical profile in comparison to Dhakhiliya and Dhahira regions in which they have a more severe manifestation of the disease and this could be explained by the Benin and CAR haplotypes in these regions respectively (Figure 2). Only one haplotype combination was found among patients from Musandam (CAR/Asian) and this can be due to the isolation of this region from the rest of the country by mountains and the UAE. SCD is absent in Wusta and Dhofar due to low levels of malaria in the past in these two regions. ${ }^{20}$ The 4 identified haplotypes, which we have referred to as 0manI, II, III and IV are expected in an admixed population such as Oman and might have been derived differently by recombinant events. ${ }^{21}$ OmanI could have probably been derived from CAR with a mutation at F5. It is also possible to say that it is a result from a recombination event between CAR and Cameroon haplotypes but this is less likely as Cameroon haplotype was not found in the Omani population. Oman II could be a result of a recombinant event between Benin and CAR while OmanIII could be the outcome of a recombination between Asian and CAR haplotypes. Oman IV could be a derivative of OmanII with a mutation at F1. However, these are just assumptions and further studies are eventually required to track back the origin of each haplotype.

\section{Early diagnosis, haplotype, hemo- globin $\mathrm{F}$ and prognosis}

Identifying the disease at an early stage and defining genotype and haplotype allows clinicians to predict to some extent the prognosis and to plan a tailored treatment. Early prediction of the clinical expression will help in preventing or reducing acute painful episodes (crisis) in this cohort, which is the most common traumatic experience in SCD, ${ }^{22}$ and ACS, which is the most common cause of death in Omani SCD patients. ${ }^{23}$

The Asian haplotype was associated with highest HbF levels, fewer hospitalizations and painful episodes and patients did not develop acute chest syndrome although vaso-occlusive events did occur. Our study also confirms that the CAR haplotype whether homozygous or combined heterozygous is associated with lowest $\mathrm{HbF}$ level and the highest incidence of organ damage and renal failure as reported elsewhere. ${ }^{24,25}$

Carriers of the $\mathrm{HbS}$ gene on the Asian haplotype on one chromosome and OmanI haplotype on the other presented in our cohort high HbF levels (average 14.2\%) and a milder clinical course than other compound heterozygous haplotypes. Bakioglu et al. ${ }^{26}$ reported mild SCD cases of Asian/Benin haplotype with high levels of $\mathrm{Hb} \mathrm{F}$ (average 22.2\%). This shows that carrying the $\mathrm{HbS}$ mutation on an Asian haplotype on one chromosome could still contribute to elevating the $\mathrm{HbF}$ expression.

Higher concentrations of $\mathrm{HbF}$ in the cell lead to lower concentrations of $\mathrm{HbS},{ }^{27}$ better oxygenation and less clinical severity. A potential threshold of $20 \% \mathrm{HbF}$ has been suggested to effectively prevent recurrent vasoocclusive episodes. ${ }^{28}$ This is true in most cases, however, in another study, some patients with HbF levels near $20 \%$ had a devastating disease manifestation. ${ }^{22}$ The same observation was seen in one of the patients in our cohort, with the Asian/CAR haplotype. Despite the high $\mathrm{HbF}$ (19.7\%), the patient is frequently admitted to the hospital with vaso-occlusive crisis, dactilitis and severe abdominal pain. This patient has no iron overload and is heterozygous for the $\alpha-3.7$ deletion. This finding imply that other circumstantial or genetic factors or external transacting determinants such as blood viscosity, elevated packed cell volume, vascular adherence, acidosis and dehydration as well as patient's life style and diet might contend with the beneficial effect of the high $\mathrm{HbF}$ level. Our findings support the conclusion by Acquaye et al. ${ }^{29}$ and Seltzer et al..$^{30}$ that fetal hemoglobin levels are very important but not the only parameters that mitigate the severity of the disease and we are at the moment inquiring which other factors could be associated with the severity of this case.

\section{Sub-haplotype}

The sub-haplotype study of 42 SNP's on the 11 haplotype combinations observed in our 125 SCD patients revealed that 15 different positions differentiate the 11 identified haplotypes. No sub-haplotypes were determined except in patients with the CAR/0manI haplotype, which revealed variation at the G- $\gamma$ promoter SNP-1 giving 3 different sub-haplotypes (Table 6). One patient with sub-haplotype-c who presented with homozygosity for the wild type 6 nucleotide sequence (CTTTAA) at the G$\gamma$ promoter, a very severe phenotype and not responding to high doses of hydroxyurea treatment. This element could be the cause of low $\mathrm{HbF}$ expression and of non-response to hydroxyurea and this hypothesis seems to be sustained by the fact that another patient with sub-haplotype-b with a homozygous mutational deletion of the 6 nucleotides at the same position became better as soon as being on the drug. The presence or absence of the $6 \mathrm{nt}$ sequence in at the $\mathrm{G}-\gamma$ promoter in the CAR/OmanI patients could be associated with the differences in clinical presentation and with response to hydroxyurea therapy, probably an element is in linkage to the 6 nt deletion and is responsible for the positive response to the drug.

\section{Conclusions}

Our study on haplotype/phenotype correlation has shown the existence of at least 11 different haplotype combinations in 0man. These are differently distributed among the six main regions of the country. Sub-haplotype was only observed in CAR/OmanI combination and could be associated with the clinical differences observed in patients with the same haplotype. Identifying haplotypes and sub-haplotypes in early life may allow a better prognosis and a more accurate risk predictions and a better-tailored therapy, to match disease-related risks and to facilitate planning of clinical trials to prevent the development of severe complications later in life. Nevertheless, we have shown that when the phenotypes are classified into; mild, intermediate and severe, neither the haplotype or the $\mathrm{HbF}$ alone appeared to be fully associable with the clinical phenotypes as also been observed by Alexander et al. ${ }^{31}$ External and/or modifying, or epistatic factors, which potentially modulate the phenotype of SCD do occur and more efforts should be done trying to chart them. The implementation of primary prevention with simple cost effective interventions for SCD are essential ${ }^{32}$ and are likely to lead to lower incidence, lower costs for public health and improved survival rate of SCD patients in Oman.

\section{References}

1. Herrick JB. Peculiar elongated and sickleshape red blood corpuscles in a case of 
severe anemia. Arch Intern Med 1910;6:517-21.

2. Geever RF, Wilson LB, Nallaseth FS, et al. Direct identification of sickle cell anemia by blot hybridization. PNAS 1981;78:50815 .

3. Ballas SK. Sickle cell pain, 2nd ed. Seattle: IASP Press; 2014.

4. Giordano PC, Huisman W, Harteveld CL. Iron depletion: an ameliorating factor for sickle cell disease? ISRN Hematol 2011;2011:473152.

5. Kulozik AE, Thein SL, Kar BC, et al. Raised $\mathrm{Hb} F$ levels in sickle cell disease are caused by a determinant linked to the $\beta$ globin gene cluster. Prog Clin Biol Res 1987;251:427-39.

6. Charache S, Terrin ML, Moore RD, et al. Effect of hydroxyurea on the frequency of painful crises in sickle cell anemia. $\mathrm{N}$ Engl J Med 1995;332:1317-22.

7. Walters MC, Patience M, Leisenring W, et al. Bone marrow transplantation for sickle cell disease. N Engl J Med 1996;335:36976 .

8. Kamel K. Heterogeneity of sickle cell anaemia in Arabs: review of cases with various amounts of fetal haemoglobin. $\mathrm{J}$ Med Genet 1979;16:428-30.

9. Pagnier J, Mears JG, Dunda-Belkhodja 0, et al. Evidence for the multicentric origin of the sickle cell hemoglobin gene in Africa. Proc Natl Acad Sci U S A 1984;81:1771-3.

10. Nagel RL, Fleming AF. Genetic epidemiology of the $\beta$ s gene. Baillieres Clin Haematol 1992;5:331-65.

11. Padmos MA, Roberts GT, Sackey K, et al. Two different forms of homozygous sickle cell disease occur in Saudi Arabia. Br J Haematol 1991;79:93-8.

12. Phylipsen M, Yamsri S, Treffers E, et al. Non-invasive prenatal diagnosis of $\beta$-thalassemia and sickle-cell disease using pyrophosphorolysis-activated polymeriza- tion and melting curve analysis. Prenatal Diagnosis 2012;32:578-87.

13. Van Delft P, Lenters E, Bakker-Verweij M, et al. Evaluating five dedicated automatic devices for haemoglobinopathy diagnostics in multi-ethnic populations. Int $\mathrm{J}$ Lab Hematol 2009;31:484-95.

14. Hassan SM, Hamza N, Jaffer Al-Lawatiya F, et al. Extended molecular spectrum of $\beta$ and $\alpha$-thalassemia in Oman. Hemoglobin 2010;34:127-34

15. Montgomery J, Wittwer CT, Palais R, et al. Simultaneous mutation scanning and genotyping by high-resolution DNA melting analysis. Nature Protocoles 2007;2:5966 .

16. Ririe KM, Rasmussen RP, Wittwer CT. Product differentiation by analysis of DNA melting curves during the polymerase chain reaction. Anal Biochem 1997;245:154-60.

17. Steinberg MH, Hsu H, Nagel RL, et al. Gender and haplotype effects upon hematological manifestations of adult sickle cell anaemia: effects of haplotype in sickle cell anaemia. Am J Hematol 1995;48:175-81.

18. Nagel RL, Fabry ME, Pagnier J, et al. Hematologically and genetically distinct forms of sickle cell anemia in Africa: the Senegal type and the Benin type. New Eng J Med 1985;312:880-4.

19. Daar S, Hussain HM, Gravell D, et al. Genetic Epidemiology of $\mathrm{HbS}$ in Oman: Multicentric Origin for the bS Gene. Am J Hematol 2000;64:39-46.

20. Rajab A, Patton MA. Major factors determining the frequencies of hemoglobinopathies in Oman. Am J Med Genet 1997;71:240-2.

21. Zago MA, Silva WA, Gualandro S, et al. Rearrangements of the $\beta$-globin gene cluster in apparently typical $\beta S$ haplotypes. Haematologica 2001;86:142-5.

22. Steinberg MH. Predicting clinical severity in sickle cell anaemia. Br J Haematol 2005;129:465-81.

23. Al Lamki L. Deaths from sickle cell disease in intensive care units. Sultan Qaboos Univ Med J 2012;12:133-6.

24. Bakanay SM., Dainer E, Clair B, et al. Mortality in sickle cell patients on hydroxyurea therapy. Blood 2005;105:545-7.

25. Nagel RL, Steinberg MH. Genetics of the bS gene: origins, epidemiology, and epistasis. In: Steinberg MH, Forget BG, Higgs DR, RL Nagel, eds. Disorders of hemoglobin: genetics, pathophysiology, and clinical management. Cambridge: Cambridge University Press; 2001. pp 711-755.

26. Bakioglu I, Hattori Y, Kutlar A, et al. Five adults with mild sickle cell anaemia share a bS chromosome with the same haplotype. Am J Hematol 1985;20:297-300.

27. Bailey K, Morris JS, Thomas P, et al. Fetal haemoglobin and early manifestations of homozygous sickle cell disease. Arch Dis Child 1992;67:517-20.

28. Powars DR, Weiss JN, Chan LS, et al. Is there a threshold level of fetal hemoglobin that ameliorates morbidity in sickle cell anemia? Blood 1984;63:921-6.

29. Acquaye JK, Omer A, Ganeshaguru K, et al. Non-benign sickle cell anaemia in western Saudi Arabia. Br J Haematol 1985;60:99108.

30. Seltzer WK, Abshire TC, Lane PA, et al. Molecular genetic studies in black families with sickle cell anemia and unusually high levels of fetal hemoglobin. Hemoglobin 1992;16:363-77.

31. Alexander N, Higgs D, Dover G, et al. Are there clinical phenotypes of homozygous sickle cell disease? $\mathrm{Br} \mathrm{J}$ Haematol 2004;126: 606-11.

32. Giordano PC. Prospective and retrospective primary prevention of hemoglobinopathies in multiethnic societies. Clin Biochem 2009;42:1757-66. 